\title{
An Explicit MOT Scheme for Solving the Nyström-Discretized TD-MFIE
}

\author{
Rui Chen and Hakan Bagci \\ Computer, Electrical and Mathematical Science and Engineering Division \\ King Abdullah University of Science and Technology (KAUST), Thuwal, 23955-6900, Saudi Arabia \\ e-mails: \{rui.chen, hakan.bagci\}@kaust.edu.sa
}

\begin{abstract}
A fully explicit marching-on-in-time scheme for solving the time domain magnetic field integral equation is proposed. The unknown current density induced on the surface of the scatterer is expanded using a higher-order Nyström method in space and Lagrange interpolation in time. The resulting system is cast in the form of an ordinary differential equation and integrated in time using a predictor-corrector scheme to obtain the unknown expansion coefficients. Numerical results demonstrate that the proposed explicit scheme can use the same time step size as its implicit counterpart without sacrificing stability and is five times faster under low-frequency excitation (i.e., for large time step).
\end{abstract}

\section{INTRODUCTION}

For solving wideband electromagnetic scattering problems, methods capable of transient analysis are preferred over frequency domain methods. Surface integral equation (SIE)based transient simulators usually employ marching-on-intime (MOT) schemes to solve for unknown equivalent currents introduced on the surface of the scatterers [1]. Depending on the types of spatial and temporal basis functions used for expanding these currents and the time step size, these MOT schemes can be either implicit or explicit [2]. Even though explicit schemes do not require solving a matrix equation at every time step, they need smaller time step sizes to guarantee stable solutions, which results in longer simulation times. To overcome these drawbacks, a quasi-explicit MOT scheme has been proposed to solve the time domain magnetic field integral equation (TD-MFIE) [2]. This solver uses Rao-WiltonGlisson (RWG) basis functions [3] to expand the surface current density in space. The semi-discretized TD-MFIE is cast into a system of ordinary differential equations (ODEs) and integrated in time using a predictor-corrector scheme to obtain the unknown expansion coefficients. The time step size required by this solver to obtain a stable solution is as large as that would be used by its implicit counterpart. The quasiexplicit MOT scheme requires the solution of a system with a (sparse) Gram matrix at every time step. Even though the sparseness of the Gram matrix does not change with the time step size, which renders this MOT scheme more efficient than its implicit counterpart under low-frequency excitation, there is still need to solve a sparse matrix system at every time step.

In this work, a fully explicit MOT solver, which does not suffer from this drawback, is proposed for solving the TDMFIE. Unlike the above quasi-explicit solver, this scheme uses a higher-order Nyström method [4] instead of RWG basis functions. Since the Nyström method uses higher-order vector basis functions defined at a set of discrete points to expand the unknown current density and point testing, the associated the Gram matrix and its inverse are block diagonal with twoby-two blocks. As a result "matrix inversion" is replaced by a single blocked-diagonal matrix-vector multiplication and the efficiency of time marching is improved significantly. Indeed, numerical results demonstrate the proposed fully explicit MOT scheme is five times faster than its implicit counterpart under low-frequency excitation (i.e., for large time step).

\section{Formulation}

Let $S$ denote the surface of a perfect electrically conducting (PEC) scatterer residing in an unbounded background medium with permeability $\mu_{0}$ and permittivity $\varepsilon_{0}$. The scatterer is excited by a band-limited incident magnetic field $\mathbf{H}^{\text {inc }}(\mathbf{r}, t)$, that is vanishingly small for $t \leq 0$ and $\forall \mathbf{r} \in S$. The surface current density $\mathbf{J}(\mathbf{r}, t)$, which is induced on $S$, is related to $\mathbf{H}^{\text {inc }}(\mathbf{r}, t)$ through the TD-MFIE:

$$
\begin{aligned}
\frac{1}{2} \partial_{t} \mathbf{J}(\mathbf{r}, t) & =\hat{\mathbf{n}}(\mathbf{r}) \times \partial_{t} \mathbf{H}^{\mathrm{inc}}(\mathbf{r}, t) \\
& +\hat{\mathbf{n}}(\mathbf{r}) \times \frac{1}{4 \pi} \int_{S} \nabla \times \frac{\partial_{t} \mathbf{J}\left(\mathbf{r}^{\prime}, t-R / c_{0}\right)}{R} d \mathbf{r}^{\prime} .
\end{aligned}
$$

Here, $R=\left|\mathbf{r}-\mathbf{r}^{\prime}\right|$ is the distance between observation and source points $\mathbf{r}$ and $\mathbf{r}^{\prime}, \hat{\mathbf{n}}(\mathbf{r})$ is the outward unit normal vector, and $c_{0}=1 / \sqrt{\mu_{0} \varepsilon_{0}}$ is the speed of light in the background medium. To numerically solve (1), first $S$ is discretized into $N_{\mathrm{p}}$ curvilinear triangular patches and $\mathbf{J}(\mathbf{r}, t)$ is expanded in space as [4]

$$
\begin{array}{r}
\mathbf{J}(\mathbf{r}, t)=\sum_{p=1}^{N_{\mathrm{p}}} \sum_{i=1}^{N_{\mathrm{i}}} \vartheta^{-1}(\mathbf{r}) L_{(i, p)}(\mathbf{r}) \\
{\left[\{\mathbf{I}(t)\}_{(i, p)}^{u} \mathbf{u}(\mathbf{r})+\{\mathbf{I}(t)\}_{(i, p)}^{v} \mathbf{v}(\mathbf{r})\right]}
\end{array}
$$

where $N_{\mathrm{i}}$ is the number of interpolation points on each patch, $\vartheta(\mathbf{r})$ is the Jacobian, $L_{(i, p)}(\mathbf{r})$ is the Lagrange interpolator defined at $\mathbf{r}_{(i, p)}$ (interpolation point $i$ on patch $p$ ), and $\{\mathbf{I}(t)\}_{(i, p)}^{u}$ and $\{\mathbf{I}(t)\}_{(i, p)}^{v}$ are the time-dependent unknown coefficient of $\mathbf{u}(\mathbf{r})$ - and $\mathbf{v}(\mathbf{r})$-directed current densities at $\mathbf{r}_{(i, p)}$. Here, $\mathbf{u}(\mathbf{r})$ and $\mathbf{v}(\mathbf{r})$ are vectors tangential to the surface of patch $p$. Inserting (2) into (1) and applying the point testing to the resulting equation at $\mathbf{r}_{(j, q)}, j=1, . ., N_{\mathrm{i}}, q=1, . ., N_{\mathrm{p}}$, yield a time-dependent linear matrix system. Sampling this system in time at $t=k \Delta t$, where $\Delta t$ is the time step, and 
using Lagrange polynomial functions $T(t)$ [5] to facilitate the computation of retarded time integrals yield

$$
\mathbf{G} \dot{\mathbf{I}}_{k}=\mathbf{V}_{k}^{\mathrm{inc}}+\sum_{l=1}^{k} \mathbf{Z}_{k-l} \mathbf{I}_{l}, k=1, \ldots, N_{\mathrm{t}} .
$$

Here, $\left\{\mathbf{I}_{l}\right\}_{(i, p)}^{b}=\{\mathbf{I}(l \Delta t)\}_{(i, p)}^{b},\left\{\dot{\mathbf{I}}_{k}\right\}_{(i, p)}^{b}=\left\{\partial_{t} \mathbf{I}(k \Delta t)\right\}_{(i, p)}^{b}$, and the entries of the blocks of $\mathbf{G}, \mathbf{V}_{k}^{\text {inc }}, \mathbf{Z}_{k-l}$ are given by

$$
\begin{aligned}
& \quad\{\mathbf{G}\}_{(j, q)(i, p)}^{a b}=\frac{1}{2} \mathbf{a}\left(\mathbf{r}_{(j, q)}\right) \cdot \mathbf{b}\left(\mathbf{r}_{(i, p)}\right) \vartheta^{-1}\left(\mathbf{r}_{(j, q)}\right) \delta_{i j} \delta_{p q} \\
& \left\{\mathbf{V}_{k}^{\mathrm{inc}}\right\}_{(j, q)}^{a}=\mathbf{a}\left(\mathbf{r}_{(j, q)}\right) \cdot \hat{\mathbf{n}}\left(\mathbf{r}_{(j, q)}\right) \times \partial_{t} \mathbf{H}^{\mathrm{inc}}\left(\mathbf{r}_{(j, q)}, k \Delta t\right) \\
& \left\{\mathbf{Z}_{k-l}\right\}_{(j, q)(i, p)}^{a b}=-\mathbf{a}\left(\mathbf{r}_{(j, q)}\right) \cdot \hat{\mathbf{n}}\left(\mathbf{r}_{(j, q)}\right) \\
& \times \frac{1}{4 \pi} \int_{\Delta p} \frac{\mathbf{R}}{R^{3}}\left[\partial_{t} T_{l}\left(k \Delta t-R / c_{0}\right)+\frac{R}{c_{0}} \partial_{t}^{2} T_{l}\left(k \Delta t-R / c_{0}\right)\right] \\
& \times \vartheta^{-1}\left(\mathbf{r}^{\prime}\right) L_{(i, p)}\left(\mathbf{r}^{\prime}\right) \mathbf{b}\left(\mathbf{r}^{\prime}\right) d \mathbf{r}^{\prime}
\end{aligned}
$$

where $a, b \in\{u, v\}$ and $\mathbf{a}(\mathbf{r}), \mathbf{b}(\mathbf{r}) \in\{\mathbf{u}(\mathbf{r}), \mathbf{v}(\mathbf{r})\}, \mathbf{R}=$ $\mathbf{r}_{(j, q)}-\mathbf{r}^{\prime}, R=|\mathbf{R}|$, and $T_{l}(t)=T(t-l \Delta t)$. The ODE system (3) is integrated in time using a $\mathrm{PE}(\mathrm{CE})^{\mathrm{m}}$ method [2] to obtain unknown current expansion coefficients. The resulting MOT scheme calls for solution of (3) at every time step, however, since $\mathbf{G}$ is block diagonal, this operation does not require any matrix inversion scheme or an iterative solver. Consequently, the efficiency of the solver is increased especially at low frequencies in comparison to its implicit counterpart.

\section{NumeriCAl Results}

The explicit MOT scheme and its implicit counterpart are used for analyzing transient electromagnetic scattering from a unit PEC sphere centered at the origin. The incident field is a Gaussian modulated wave $\mathbf{H}^{\text {inc }}(\mathbf{r}, t)=\hat{y} \sqrt{\varepsilon_{0} / \mu_{0}} G\left(t-t_{\mathrm{p}}-\right.$ $\left.z / c_{0}\right)$, where $G(t)=\cos \left(2 \pi f_{0} t\right) e^{-t^{2} / 2 \sigma^{2}}, \sigma=7 /\left(2 \pi f_{\mathrm{bw}}\right)$, and $t_{\mathrm{p}}=3.5 \sigma+10 /\left(f_{0}+f_{\mathrm{bw}}\right)$. Here, $f_{0}$ is modulation frequency and $f_{\mathrm{bw}}$ is the bandwidth. In all simulations, a second-order Nyström method is used, $T(t)$ is a third-order Lagrange polynomial, and the $\mathrm{PE}(\mathrm{CE})^{\mathrm{m}}$ scheme uses fourthorder Adam-Bashforth and third-order backward differentiation formula coefficients as the predictor and corrector coefficients, respectively [6]. The implicit scheme uses a transposefree quasi-minimal residual (TFQMR) method [7] to solve the MOT system iteratively at every time step. The convergence thresholds for implicit (iterative solver) and explicit (corrector updates) schemes are both set to $10^{-13}$.

The unit sphere is discretized into $N_{\mathrm{p}}=622$ curvilinear patches, which results in 7464 unknown current coefficients. For the first simulation, $f_{0}=300 \mathrm{MHz}, f_{\mathrm{bw}}=200 \mathrm{MHz}$, $\Delta t=0.1 \mathrm{~ns}$, and $N_{\mathrm{t}}=1400$. Fig. 1 compares the magnitude of the expansion coefficient computed by the explicit and implicit schemes at point $(0.430,0.857,-0.283 \mathrm{~m})$ along the direction $(0.223,-0.050,0.186)$. The results agree very well. Additionally, the radar cross section (RCS) values computed using the Mie series and MOT solutions at $f=300 \mathrm{MHz}$ for $\theta=\left[0^{\circ}, 180^{\circ}\right]$ and $\phi=0^{\circ}$ are compared in Fig. 2. The figure clearly shows the accuracy of the proposed solver. For the second simulation, $f_{0}=5 \mathrm{MHz}, f_{\mathrm{bw}}=3.75 \mathrm{MHz}$,
$\Delta t=11.429 \mathrm{~ns}$, and $N_{\mathrm{t}}=500$. This is a low frequency (large $\Delta t$ ) problem, where the implicit scheme has to solve a full matrix system at every time step. Therefore, the proposed explicit scheme is 5.35 times faster.

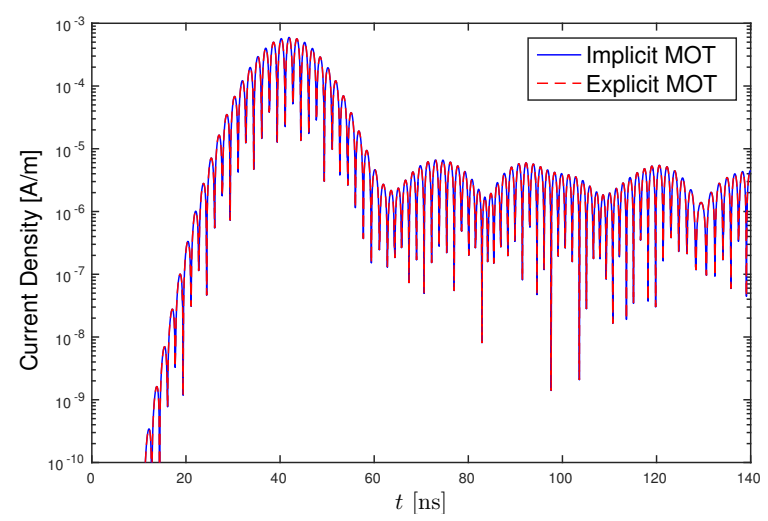

Fig. 1. Current coefficients computed by the MOT schemes.

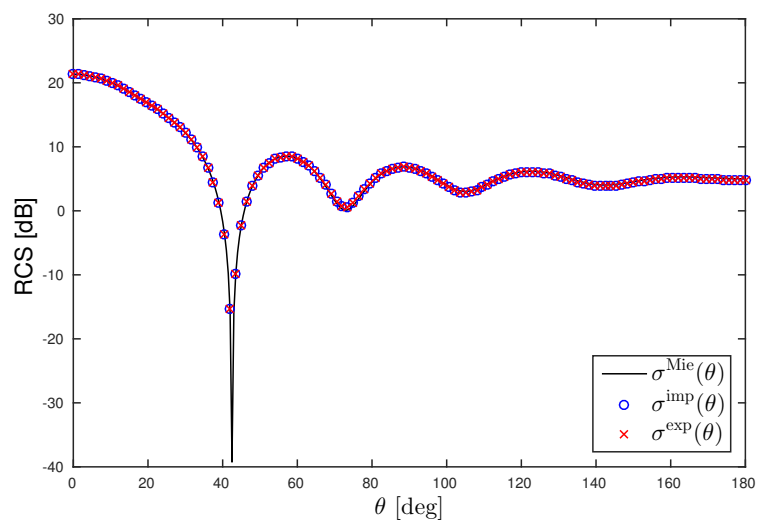

Fig. 2. RCS computed using the Mie series and MOT solutions.

\section{REFERENCES}

[1] B. Shanker, A. A. Ergin, K. Aygun, and E. Michielssen, "Analysis of transient electromagnetic scattering from closed surfaces using a combined field integral equation", IEEE Trans. Antennas Propag., vol. 48, no. 7, pp. 1064-1074, July 2000.

[2] H. A. Ulku, H. Bagci, and E. Michielssen, "Marching on-in-time solution of the time domain magnetic field integral equation using a predictorcorrector scheme", IEEE Trans. Antennas Propag., vol. 61, no. 8, pp. 4120-4131, Aug. 2013

[3] S. M. Rao, D. R. Wilton, and A. W. Glisson, "Electromagnetic scattering by surfaces of arbitrary shape", IEEE Trans. Antennas Propag., vol. 30, no. 3, pp. 408-418, May. 1982.

[4] G. Kang, J. M. Song, W. C. Chew, K. C. Donepudi, and J. M. Jin, "A novel grid-robust higher order vector basis function for the method of moments", IEEE Trans. Antennas Propag., vol. 49, no. 6, pp. 908-915, Jun. 2001.

[5] H. Bagci, A. E. Yilmaz, V. Lomakin, and E. Michielssen, "Fast solution of mixed-potential time-domain integral equations for half-space environments", IEEE Trans. Geosci. Remote Sens., vol. 43, no. 2, pp. 269-279, Feb. 2005.

[6] E. Hairer, S. P. Norsett, and G. Warner, Solving Ordinary Differential Equations I: Nonstiff Problems, 2nd rev. ed. New York, NY, USA: Springer, 2010.

[7] R. W. Freund, "A transpose-free quasi-minimal residual algorithm for non-Hermitian linear systems", SIAM J. Sci. Comput., vol. 14, no. 2, pp. 470-482, Mar. 1993. 